\title{
Blood type and pancreatic cancer
}

In the US, pancreatic cancer is the fourth leading cause of cancer-related mortality. The genetic risk factors for most patients with pancreatic cancer are unknown, although Wolpin and colleagues have discovered an association between $\mathrm{ABO}$ blood group and the risk of pancreatic cancer. Specifically, they found that patients with blood type $\mathrm{A}, \mathrm{AB}$ or $\mathrm{B}$ were more likely to develop the disease.

Previous small, retrospective studies have hinted at a link between inherited blood group antigens and risk of developing pancreatic cancer. In this study, the researchers used two large, independent, prospective cohort studies to examine the relationship between $\mathrm{ABO}$ blood type and risk of pancreatic cancer. Data were collected on a total of 107,503 US health professionals, and 316 of these developed pancreatic cancer during follow-up. The relationship between blood type and risk of pancreatic cancer was very similar in the two cohorts. In all, $17 \%$ of pancreatic cancer cases were linked to inheriting blood group A, B or $\mathrm{AB}$. Patients with blood group $\mathrm{O}$ had the lowest age-adjusted incidence rate for pancreatic cancer. Other risk factors such as age, smoking status, BMI and physical activity were not significantly associated with an increased risk of the effect of blood group on pancreatic cancer.

Further studies are needed to establish the mechanisms by which ABO subtypes or closely linked genetic variations influence the risk of pancreatic cancer, and to determine the biological basis for this association.

Mandy Aujla

Original article Wolpin, B. M. et al. ABO blood group and the risk of pancreatic cancer. J. Natl Cancer Inst. 101, 424-431 (2009). 